\title{
Artigo Original / Original Paper \\ Helechos y Licofitas de la Reserva de Biosfera Yabotí, Misiones, Argentina: Parque Provincial Moconá
}

Ferns and Lycophytes from Yabotí Biosphere Reserve, Misiones, Argentina: Parque Provincial Moconá

\author{
Santiago J. Kelly ${ }^{1,4}$, Agustina Yañez ${ }^{2,4,5,6}$, Daniela Ocampo Terraza ${ }^{3}$ \& Gonzalo J. Marquez ${ }^{3,4}$
}

\begin{abstract}
Resumen
E1 Parque Provincial Moconá se encuentra situado en el extremo este de la provincia de Misiones (Argentina), circundado por los ríos Yabotí y Uruguay. Pertenece a la Reserva de la Biosfera Yabotí y, en conjunto con otras áreas protegidas dentro de la Reserva Yabotí, representa uno de los principales relictos de selva paranaense del país. En el presente trabajo se llevó a cabo un relevamiento exhaustivo de las especies de helechos y licofitas que crecen en el parque. Se realizaron viajes de recolección al área de estudio y se consultaron los herbarios nacionales. Se hallaron 71 taxones de los cuales 38 representaron nuevos registros para el área protegida. Se provee una clave para el reconocimiento de los taxones y se realizan consideraciones respecto a su hábitat. Adicionalmente se discute su relación con el Parque Estadual do Turvo, Rio Grande do Sul (Brasil) con el cual limita, y la relevancia respecto a la conservación de la flora nativa.
\end{abstract}

Palabras clave: biodiversidad, Paranaense, Reservas de la Biosfera, Río Uruguay.

\begin{abstract}
The Parque Provincial Moconá is in the east of the Misiones province, surrounded by Yabotí and Uruguay rivers. It belongs to Yabotí Biosphere Reserve, and together with other Biosphere's protected areas, it represents one of the main relicts of the Paranaense forest in the country. In this work, fern and lycophyte species that grow in the park were exhaustively surveyed. Field trips were carried out to the study area and national herbaria were consulted. Seventy-one taxa were found, 38 of which represent new records for the protected area. A comprehensive key to the species and habitat considerations are provided. Additionally, the relationship with the adjacent protected area Parque Estadual do Turvo, Rio Grande do Sul (Brazil) and the relevance to the conservation of native flora is discussed.
\end{abstract}

Key words: biodiversity, Paranaense, Biosphere Reserves, Uruguay River.

\section{Introducción}

El Parque Provincial Moconá $\left(27^{\circ} 08^{\prime} 35^{\prime}\right.$ 'S, $53^{\circ} 53^{\prime} 12^{\prime \prime}$ ), se ubica en el extremo sur del Departamento de San Pedro, Misiones, Argentina (Fig. 1) y comprende un área de 999 ha de selva subtropical mixta circundada por los ríos Yabotí y
Uruguay (Bertolini 1999). Se encuentra dentro del distrito de las Selvas, de la provincia Paranaense, en el esquema de Cabrera \& Willink (1973) y la provincia del Bosque Paranaense, subregión Paranaense, según Morrone (2001). Asimismo, forma parte del sistema de áreas protegidas de

\footnotetext{
${ }^{1}$ Universidad Nacional de La Plata (UNLP)-CCT La Plata, CONICET, Inst. Fisiología Vegetal (INFIVE), Diagonal 113 esq. 61, B1900, La Plata, Argentina.

${ }^{2}$ Museo Argentino de Ciencias Naturales "Bernardino Rivadavia", CONICET, División Plantas Vasculares, Av. Ángel Gallardo 470, Piso 2, C1405DJR, Ciudad Autónoma de Buenos Aires, Argentina.

${ }^{3}$ Universidad Nacional de La Plata, Facultad de Ciencias Naturales y Museo, Cátedra de Palinología, Calle 643 esq. 120, B1900, La Plata, Buenos Aires, Argentina.

${ }^{4}$ Consejo Nacional de Investigaciones Científicas y Tecnológicas (CONICET), Godoy Cruz 2290, C1425FQB, CABA, Argentina.

${ }^{5}$ ORCID: <https://orcid.org/0000-0002-4508-2148>

${ }^{6}$ Autor para correspondencia: gugu@macn.gov.ar
} 


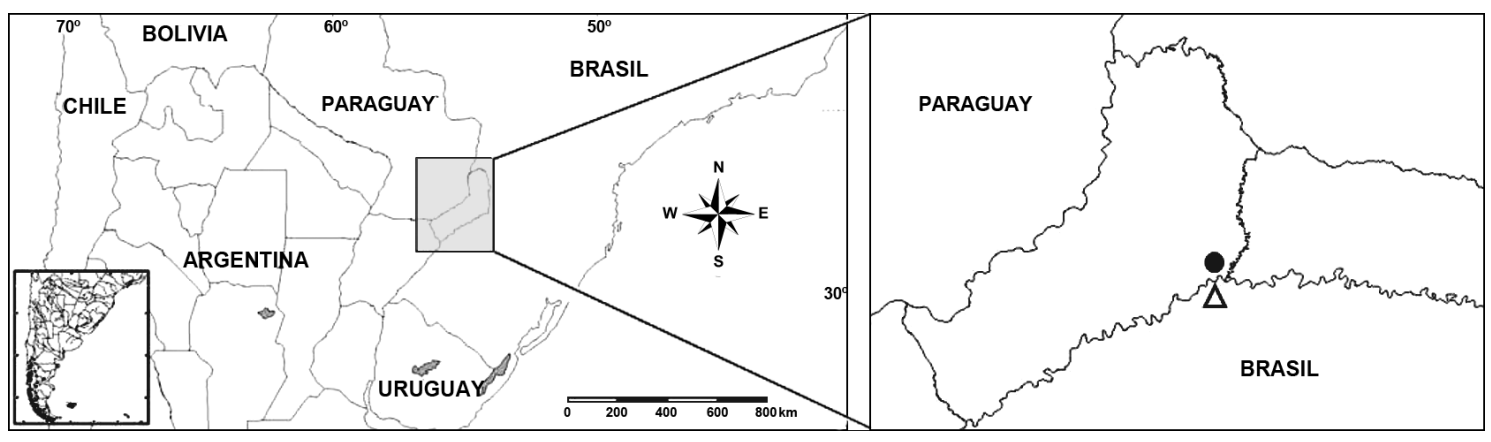

Figura 1 - Localización del Parque Provincial Moconá, Misiones, Argentina. ( $\bullet$ Parque Provincial Moconá; $\Delta=$ Parque Estadual do Turvo).

Figure 1 -Location of Parque Provincial Moconá Provincial, Misiones Argentina. ( $=$ Parque Provincial Moconá. $\Delta=$ Parque Estadual do Turvo).

la Reserva de Biosfera Yabotí, junto con el Parque Provincial Esmeralda, el Parque Provincial Caá Yarí, La Reserva Forestal Guaraní y la Reserva Natural Cultural Papel Misionero.

Debido a que el Parque Moconá limita con el Parque Estadual do Turvo (Rio Grande do Sul, República Federativa del Brasil), constituye un nexo entre áreas destinadas a la conservación de fauna y flora de Argentina y Brasil y se considera una zona fundamental en la conservación de la biodiversidad de la selva subtropical.

La Subregión Paranaense es un importante centro de diversidad vegetal que se ve reflejado en el elevado número de estudios florísticos realizados hasta el momento en el área (Bolòs et al. 1991; Peña-Chocarro et al. 1999; Borgo \& Menezes-Silva 2003; Sobral et al. 2006; Céspedes \& Mereles 2006; Schwartsburd \& Labiak 2007; Inacio \& Jarenkow 2008; Mynssen 2011; Kuhnen et al. 2015). A pesar de ello, en la provincia de Misiones existen escasos trabajos de este tipo, entre los que se pueden destacar los relevamiento de la flora del sur de Misiones (Fontana 1998), del Parque Provincial Teyú Cuaré (Biganzoli \& Múlgura de Romero 2004) y de la Reserva de usos Múltiples Guaraní (Tressens et al. 2008).

Particularmente en el caso de los Helechos y las Licofitas, a pesar de que el Nordeste de Argentina ha sido registrado como un centro de mayor diversidad de estos grupos (Ponce et al. 2002), en Misiones (Argentina), solamente se puede citar el trabajo florístico de Marquez et al. (2006) en la Reserva "Valle del Arroyo Cuñá Pirú", departamento de Cainguás.

El Parque Provincial Moconá en particular, posee un inventario preliminar de especies de plantas vasculares y no vasculares (Daviña et al. 1999, 2003; Coccoza Espósito 2004), con adiciones posteriores en lo que respecta a helechos (Yañez et al. 2011; Marquez et al. 2016).

El objetivo de este trabajo fue llevar a cabo un relevamiento florístico exhaustivo de los helechos y licofitas del Parque Provincial Moconá. Asimismo se proporcionan datos respecto al hábitat, los cuales aportarán información a la discusión sobre la importancia de estos grupos en la conservación de áreas protegidas. Adicionalmente, se brinda una clave para su reconocimiento.

Este trabajo representa la primera contribución a un proyecto mayor que pretende profundizar el conocimiento de los Helechos y Licofitas presentes en el sistema de áreas protegidas que conforman la Reserva de Biósfera Yabotí.

\section{Materiales y Métodos}

Los ejemplares utilizados en este trabajo provienen de recolecciones realizadas entre los años 2007 y 2015 al Parque Provincial Moconá. El material fue depositado en el herbario LP, previa herborización mediante las técnicas clásicas. Asimismo, se utilizaron ejemplares depositados en los herbarios BA, CTES, G, LP, MNES y SI para comparar y ampliar la información obtenida en campo.

Para la determinación del material se utilizaron claves y descripciones presentes en diversos trabajos florísticos de Argentina (Sota de la 1977; Ponce 1994; Ponce \& Arana 2016), Brasil (Sehnem 1968, 1972, 1979), Perú (Tryon \& Stolze 1989, 1991) y Mesoamerica (Moran 1995). También se utilizaron relevamientos realizados en otras áreas protegidas de la provincia de Misiones, como los de Biganzoli \& Múlgura de Romero (2004), Marquez et al. (2006), Tressens et al. (2008) y revisiones de familias y géneros halladas para el área de estudio (Sota de la 1960; Sota de la \& Mickel 1969; Ponce 1987, 1998; 
Ponce et al. 2012; Giudice 1999; Hirai \& Prado 2000, 2012; Prado \& Windisch 2000; Martínez 2003; Prado 2004; Sakagami 2006; Prado \& Hirai 2008, 2010; Moran et al. 2009; Costa Assis \& Salino 2011; Arana 2012). Se siguió al PPG I (2016) para la designación taxonómica de los grupos.

\section{Resultados}

Como resultado de este trabajo se enumeran 67 taxones, pertenecientes a 38 géneros y 16 familias de helechos, y cuatro taxones, pertenecientes a dos géneros y dos familias de licofitas que crecen en el Parque Provincial Moconá. La cita completa de las especies y taxones infraespecíficos y sus familias; la información sobre los ambientes y el hábito de cada una, se presentan en la Tabla S1 (disponible en material complementario <https:// doi.org/10.6084/m9.figshare.9887627.v1>). Las familias mejor representadas en cuanto al número de géneros y especies fueron Pteridaceae (siete y 17 respectivamente) y Polypodiaceae (siete y 13 respectivamente). Asimismo, fue importante el número de familias con un único representante (Fig. 2).

En relación al hábito de las especies halladas, el $80 \%$ resultaron ser terrestres o saxícolas mientras que sólo el $20 \%$ presentaron un hábito epífito. No obstante, Lomaridium acutum (Desv.) Gasper \& V.A.O.Dittrich, Asplenium clausenii Hieron., Asplenium inaequilaterale Willd., Asplenium uniseriale Raddi y Didymochlaena truncatula (Sw.) J.Sm. se observaron creciendo tanto sobre sustrato inerte como sobre otras especies de plantas; particularmente, sobre los rizomas arborescentes de Alsophila setosa Kaulf.

Si bien el ambiente donde crecen la mayoría de los helechos y licofitas fue el interior de la selva primaria o secundaria, algunas especies se encontraron en otros hábitats. En este sentido, Osmunda spectabilis Willd., Psilotum nudum (L.) P.Beauv. y Cyclosorus interruptus (Willd.) H.Itô crecen en lagunas, donde el suelo se encuentra permanentemente inundado y se observan grandes gaps en la vegetación arbórea, por donde la luz del sol alcanza los estratos más bajos de la selva. Otro ambiente particular se observó en los márgenes expuestos del río Uruguay, donde se hallaron poblaciones de Adiantum latifolium Lam., Doryopteris triphylla (Lam.) Christ, y Lygodium venustum Sw., las cuales crecen en los espacios húmedos existentes entre las rocas. En el último caso, asimismo, adquieren un hábito trepador sobre el arbustal de la costa. Sobre las rocas, también se halló Selaginella marginata (Humb. \& Bonpl. ex Willd.) Spring.

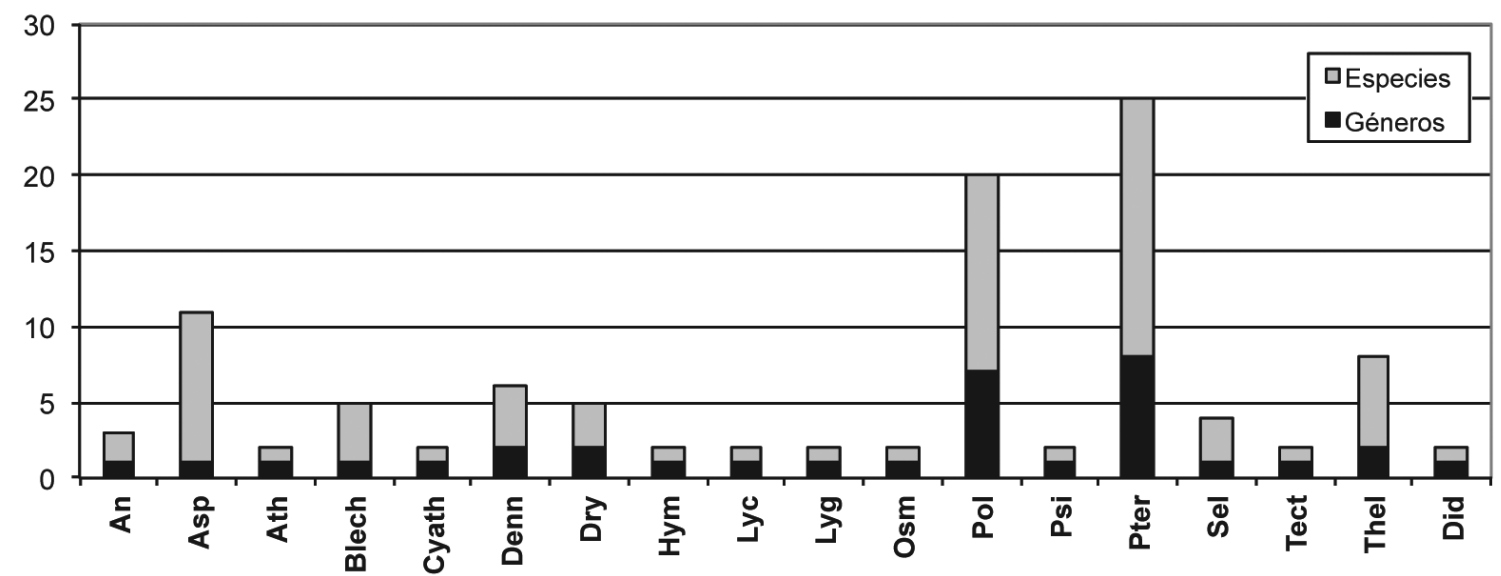

Figura 2 - Familias de helechos y licofitas presentes en el Parque Provincial Moconá. En negro se muestra el número de géneros y en gris el número de especies pertenecientes a cada familia. Familias: An = Anemiaceae; Asp = Aspleniaceae; Ath $=$ Athyriaceae; Blechnaceae $=$ Blech; Cyath = Cyatheaceae; Denn = Dennstaedtiaceae; Did = Didymochlaenaceae; Dry = Dryopteridaceae Hym = Hymenophyllaceae Lyc $=$ Lycopodiaceae Lyg = Lygodiaceae Osm = Osmundaceae Pol = Polypodiaceae $;$ Psi = Psilotaceae Pter $=$ Pteridaceae Selaginellaceae $=$ Sel Tect $=$ Tectariaceae; Thel $=$ Thelypteridaceae . Figure 2 - Ferns and lycophytes families from Parque Provincial Moconá. In black, number of genera and in gray number of species shown for each family. Families: An = Anemiaceae; Asp = Aspleniaceae; Ath = Athiriaceae; Blechnaceae = Blech; Cyath $=$ Cyatheaceae; Denn $=$ Dennstaedtiaceae; Did = Didymochlaenaceae; Dry = Dryopteridaceae; Hym = Hymenophyllaceae; Lyc = Lycopodiaceae; Lyg = Lygodiaceae; Osm $=$ Osmundaceae $; \mathrm{Pol}=$ Polypodiaceae $;$ Psi $=$ Psilotaceae Pter $=$ Pteridaceae $;$ Selaginellaceae $=$ Sel $;$ Tect $=$ Tectariaceae $;$ Thel $=$ Thelypteridaceae . 


\section{Clave de identificación de los Helechos y Licofitas del Parque Provincial Moconá}

1. Esporangios fusionados en sinangios.

Psilotum nudum

1'. Esporangios no fusionados en sinangios.

2. Expansiones laminares uninervias (licofilos); protoxilema exarco; esporangios en las axilas de los licofilos.....

2'. Expansiones laminares con vascularización compleja (eufilos); protoxilema mesarco; esporangios de posición abaxial en los eufilos.

3. Plantas isosporadas, licofilos sin lígula; rizomas sin estructuras accesorias.....

Phlegmariurus mandiocanus

3'. Plantas heterosporadas, licofilos con lígula; rizomas con rizóforos

4. Estróbilos aplanados lateralmente. 4

4'. Estróbilos tetragonales Selaginella muscosa

5. Licofilos peltados, con ápice agudo a apiculado 5

5'. Licofilos no peltados, con ápice aristado Selaginella marginata

6. Plantas con hábito arborescente, de 3-12 metros de altura; bases de los pecíolos persistentes en el rizoma Alsophila setosa

6'. Plantas con hábito herbáceo, arbustivo, epifítico o voluble; bases de los pecíolos generalmente no persistentes en el rizoma.

7. Plantas volubles Lygodium venustum

7'. Plantas herbáceas, arbustivas o epífitas 8

8. Expansión laminar con mesófilo uniestratificado excepto en la nervadura donde se pueden encontrar hasta tres capas de células...

Crepidomanes pyxidiferum

8'. Expansión laminar con mesófilo pluriestatrificadas ....................... 9

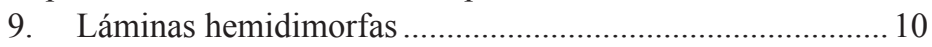

9'. Laminas isomorfas u holomorfas ........................................ 12

10. Láminas con pinnas terminales fértiles, el resto estériles; esporangio con un anillo de dehiscencia lateral formado por un grupo de cèlulas escasamente diferenciadas

Osmunda spectabilis

10'. Láminas con pinnas basales fértiles y apicales estériles; esporangios con anillo de dehiscencia apical u oblícuo, bien diferenciado 11

11. Láminas 1-pinnadas, con venación anastomosada; esporas con lomos angostos ornamentados con baculas. Anemia phyllitidis

11'. Laminas 2-pinnadas, con venación libre; esporas con lomos anchos ornamentados con espinas

Anemia tomentosa var. anthriscifolia

12. Soros o cenosoros, desnudos o protegidos por el márgen de la lámina (pseudoindusio) .......13

12'. Soros o cenosoros protegidos por verdaderos indusios, persistentes o fugaces

13. Soros o cenosoros protegidos por pseudoindusios.

13'. Soros o cenosoros desnudos 26

14. Esporangios sobre los pseudoindusios de contorno orbicular a subobircular; pínnulas cuneado-flabeladas o raramente dimidiadas. 
14'. Esporangios protegidos por el margen reflexo de la lámina, nunca sobre éste; pínnulas de otra forma

15. Frondes hasta $40 \mathrm{~cm}$ de largo; pínnulas subdimidiadas; $2-8$ soros por pseudoindusio; escamas rizomáticas con margen denticulado y base marcadamente ciliada. Adiantum latifolium

15'. Frondes mayores a $40 \mathrm{~cm}$ de largo; pínnulas flaveladas; 1 soro por pseudoindusio; escamas rizomáticas de margen entero o ciliado...

16. Raquis flexuosa; escamas rizomáticas con margen ciliado.........................Adiantum poiretti

16'. Raquis rectos; escamas rizomáticas de margen entero

17. Pínnulas con relación largo/ancho 1:1, generalmente con base cuneado-simétrica; peciólulos de 2-3 mm de largo Adiantum lorentzii

17’. Pínnulas con relación largo/ancho 2:1, generalmente con base cuneado-asimétrica; peciólulos de $0.8-1.2 \mathrm{~mm}$ de largo Adiantum raddianum

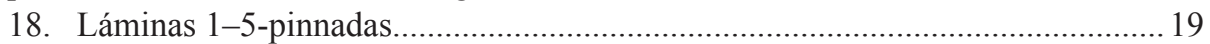

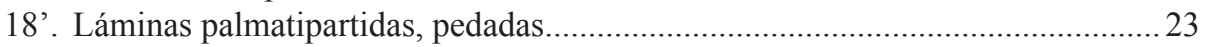

19. Esporangios agrupados en soros marginales discontinuos, o más o menos confluentes; pecíolos castaño oscuros a negros. 20

19'. Esporangios a lo largo de la vena colectora marginal (cenosoros). Pecíolos pajizos a castaños

20. Rizoma rastrero; lámina 2-3 pinnada, pinnas de disposición alterna ..... Adiantopsis chlorophylla

20'. Rizoma suberecto; lámina radiada, pinnas de disposición verticilada .... Adiantopsis radiata

21. Láminas de contorno deltoide, varias veces pinnada; nervaduras libres...... Pteris deflexa

21'. Laminas de contorno aovado a eliptico-aovado, 1-pinnadopinnatifidas; nervaduras reticuladas 22

22. Pinnas proximales con 3-7 segmentos sobre el lado basiscópico; pinnas medias pinnatifidas sobre el lado basiscópico.

..Pteris denticulada var. denticulata

22'. Pinnas proximales con 1-2 segmentos sobre el lado basiscópico; pinnas medias enteras .......... Pteris denticulada var. tristicula

23. Láminas 3-lobadas; venación libre; pecíolos teretes; vena colectora fértil submarginal Doryopteris triphylla

23'. Láminas 2-3-pinnatifidas; venación reticulada, cuando libre, con pecíolo surcado; vena colectora fértil marginal 24

24. Láminas con venación libre; pecíolo surcado con alas. Doryopteris concolor

24'. Láminas con venación anastosomada; pecíolo terete 25

25. Laminas con yemas foliares en la base; pecíolos castaño-rojizos; cenosoros continuos

Doryopteris arifolia

25'. Láminas sin yemas foliares en la base; pecíolos castaño-negruzcos; cenosoros discontinuos ....

Doryopteris pentagona

26. Esporangios creciendo sobre las venas laterales.

26'. Esporangios agrupados en soros circulares o, si lineares, a lo largo de dos venas colectoras paralelas a la vena media....29 
27. Plantas anuales, con láminas glabras

27'. Plantas perennes, con láminas pilosas o con ceras.

28. Láminas pilosas, 1-pinnadas, a veces las basales lobuladas

Hemionitis tomentosa

28'. Láminas con ceras blancas o amarillo claro en el envés, 2-pinnado-pinnatífidas.

Pityrograma calomelanos var. austroamericana

29. Soros lineales hundidos en lámina; pecíolos no articulados al rizoma Vittaria lineata

29'. Soros circulares, superficiales; pecíolos articulados al rizoma

30. Láminas enteras

30'. Laminas pinnasectas o pinnadas

31. Frondes dimorfas a subdimórfas

Microgramma squamulosa

31'. Frondes isomorfas

32. Láminas con una hilera de soros entre venas laterales.

Niphidium crassifolium

32'. Láminas con dos hileras de soros entre venas laterales 33

33. Pecíolos espaciados; nervaduras laterales flexuosas, poco o no sobresalientes en la cara abaxial de la lámina, sin venas arcuadas

Campyloneurum minus

33'. Pecíolos aproximados; nervaduras laterales rectas y paralelas, sobresalientes en la cara abaxial de la lámina; con venas arcuadas transversales a las venillas inclusas. Campyloneurum nitidum

34. Soros sin parafisos

34'. Soros protegidos por parafisos

35. Raquis con yema prolífica en el ápice

Parapolystichum effusum

35'. Raquis sin yema prolífica en el ápice 36

36. Plantas epífitas o saxícolas Phlebodium areolatum

36'. Planta terrestres.

37. Raquis de la pinna densamente pubecentes en la superficie abaxial; pelos subestrigosos

37'. Raquis de la pinna glabros o glabrecentes, pelos cuando presentes de otro tipo

Megalastrum connexum

38. Soros protegidos por escamas peltadas cuando inmaduros; láminas densamente escamosas ....

38'. Soros nunca protegidos por escamas peltadas; láminas pilosas, a veces con pocas escamas sobre los ejes, o glabras

39. Frondes menores a $12 \mathrm{~cm}$ de largo; segmentos de hasta $1 \mathrm{~cm}$ de largo.

Pleopeltis minima

39'. Frondes mayores a $12 \mathrm{~cm}$ de largo; segmentos de más de $1 \mathrm{~cm}$ de largo..... 40

40. Lámina pinnatisecta; segmentos lineares ascendentes con escasas escamas .......Pleopeltis pleopeltifolia

40'. Lamina pinnada; segmentos lineares perpendiculares al raquis, con escamas numerosas

Pleopeltis hirsutissima 
41. Rizomas con escamas peltadas; láminas gabras

41'. Rizomas con escamas basifijas; láminas pilosas

42. Raquis con escamas diminutas de hasta $1 \mathrm{~mm}$ de largo.

42'. Raquis pilosos, sin escamas

43. Venas de los segmentos simples.

43'. Venas de los segmentos furcadas

44. Láminas con base atenuada y segmentos basales auriculiformes; estípites con pelos cortos y escamas. Pecluma pectinatiforme

44'. Laminas con base subtruncada; segmentos basales menores al resto pero nunca auriculiformes; estípites con escasos pelos o glabros, sin escamas .....Pecluma venturii 45. Láminas con cenosoros .

45'. Láminas con soros de diversas formas, nunca cenosoros 50

46. Cenosoros marginales protegidos por el margen reflexo y modificado de la lámina y por un verdadero indusio interno, a veces no conspicuo.

Pteridium esculentum subpsp. arachnoideum

46'. Cenosoros protegidos por un indusio de inserción lateral.

47. Plantas hemiepífitas; expansiónes laminares dimorfas

Lomaridium acutum

47'. Plantas terrestres, expansiones laminares monomorfas 48

48. Planta con hábito semiarborescente; lámina pinnatífida

..Neoblechnum brasiliense

48'. Plantas con hábito herbáceo; lámina pinnada o pinnatisecta....... 49

49. Lámina pinnada; pinna apical bien desarrollada; $3-10$ pares de pinnas por lámina Blechnum gracile

49'. Lámina pinnatisecta; ápice de la lámina pinnatífido; 20-30 pares de pinnas por laminas. ..Blechnum occidentale

50. Soros circulares 51

50'. Soros alargados, elípticos o lineales 60

51. Soros circulares con indusio cupuliforme; rizomas con pelos 52

51'. Soros circulares con indusio nunca cupuliforme; rizoma con escamas 53

52. Rizoma con pelos catenulados; pecíolo sin raíces en la base; cóstulas bordeadas adaxialmente por un ala herbácea basiscópicamente decurrente en el eje del siguiente orden inferior

Dennstaedtia globulifera

52'. Rizoma con pelos ciliformes; pecíolo con raíces en la base; cóstulas no bordeadas adaxialmente por un ala herbácea, o si presente, nunca basiscópicamente decurrente en el eje del orden siguiente inferior... Dennstaedtia cicutaria

53. Láminas $2-3$ pinnado-pinnatífidas; nervaduras nunca extendidas hasta el margen, ensanchadas en el ápice Macrothelypteris torresiana

53'. Láminas 1 pinnado-pinnatífidas; nervaduras libres extendidas hasta el margen, no ensanchadas en el ápice o nervaduras anastomosadas 54 
54. Pínnulas basales divididas, con un lóbulo o segmento basiscópico; venación areolada..

54'. Pínnulas basales enteras, sin lóbulo o segmento basiscópico; venación libre o sólo el par de venas basales de segmentos adyacentes unidas

55. Pelos 1-celulares, pedicelados, 2-5 ramificados, presentes al menos sobre el raquis y sobre escamas del rizoma. Goniopteris scabra

55'. Si presenta pelos en el raquis o escamas del rizoma, nunca ramificados. 56 56. Rizomas suberectos; ejes y láminas escamosos Ctenitis submarginalis

56'. Rizomas rastreros o decumbentes; ejes y/o láminas pilosos 57

57. Par de venas proximales libres, alcanzando el margen por arriba del seno; esporas reticuladas o equinadas Amauropelta sanctae-catharinae

57'. Par de venas proximales unidas, conniventes o libres, alcanzando el margen justo o por debajo del seno; esporas crestadas

58. Plantas palustres; rizomas largamente rastreros, negros, sin escamas

58'. Plantas terrestres; rizomas cortamente rastreros, cubierto por escamas castañas ...

59. Pecíolos pajizos a castaño claros; costas abaxiales con pelos cortos y largos; indusios con pelos de mas de $0,3 \mathrm{~cm}$, aciculares Christella hispidula

59'. Pecíolos castaños, verdosos a atropúrpureos; costas abaxiales e indusios con pelos cortos menores de $0,2 \mathrm{~cm}$, setiformes Christella dentata 60. Láminas enteras con venación anastomosada

60'. Láminas 1-3 pinnadas con venación dicotómica abierta.....

61. Frondes subdimorfas; láminas ovadas; pecíolos de 5-20 cm de largo. Asplenium balansae

61'. Frondes monomorfas; láminas angostamente elíptico-lanceoladas; pecíolos de $1-7 \mathrm{~cm}$ de largo

62. Láminas enteras Asplenium brasiliense

62'. Láminas pinnadas o más divididas Asplenium serratum

63. Láminas pinnadas 63

63'. Láminas pinnado-pinnatífidas a 3-pinnadas 64

64. Láminas con yemas prolíferas 67 64'. Láminas sin yemas prolíferas 65

65. Plantas pequeñas, de $5-15 \mathrm{~cm}$ de alto; pinnas de $0.5-1 \times 0.2-0.4 \mathrm{~cm}$ Asplenium ulbritchii

65'. Plantas mayores, de $15-25 \mathrm{~cm}$ de alto; pinnas de 2.5-5 × 0.8-1.4 ....... Asplenium kunzeanum

66. Pinnas basales no reducidas o el par basal apenas menor; ápice de la lámina pinnatífido......Asplenium inaequilaterale

66'. Pinnas basales gradualmente reducidas; ápice de la lámina lanceolado-acuminado o romboide acuminado

Asplenium claussenii

67. Láminas pinnado-pinnatífidas......68

67'. Láminas 2-3 pinnadas 69

68. Plantas terrestres; láminas glabras; indusios a ambos lados de la vena lateral

Diplazium cristatum 
68'. Plantas epífitas o saxícolas; láminas con escamas espaciadas; indusios hacia un lado de la vena lateral Asplenium auritum

69. Láminas 2-pinnadas, frondes grandes, normalmente de $150 \mathrm{~cm}$ o más; pínnulas dimidiadas ....... Didymochlaena truncatula

69'. Láminas 3-pinnadas, frondes pequeñas, de menos de $50 \mathrm{~cm}$; pínnulas ovadas o deltoides ....... 70

70. Ápice de la fronde terminado en un segmento sin pinnas que porta una yema foliar; pinnas y pínnulas brevemente pecioluladas o subsésiles. Asplenium uniseriale

70’. Ápice de la fronde pinnado, sin yema foliar; pinnas y pínnulas largamente pecioluladas ..... Asplenium scandicinum

\section{Discusión}

Este relevamiento representa un importante aporte al conocimiento de la flora del Parque, debido a que se citaron 36 nuevos registros de helechos y 2 de licofitas para el área (Tab. S1, disponible en material complementario <https://doi.org/10.6084/ m9.figshare.9887627.v1>), respecto a los últimos informes realizados por otros autores (Daviña et al. 1999, 2003; Coccoza Espósito 2004). Si bien en el presente trabajo no se pudo acceder a los ejemplares de referencia estudiados por Daviña et al. (2003), la especie Selaginella novae-holandiae (Sw.) Spring no fue observada en los relevamientos realizados. En base a la información disponible en la Flora Argentina (Ponce \& Arana 2016) esta especie se encuentra citada solo para el noroeste del país, por lo que creemos que la mención para el Parque Provincial Moconá podría deberse a una confusión en la determinación.

De modo similar, tampoco se pudo documentar la presencia de Hymenasplenium laetum (Sw.) L.Regalado \& C.Prada, (sub Asplenium laetum Sw., Daviña et al. 2003). El especimen depositado en LP mencionado por Daviña et al. (2003) no pudo ser ubicado. Por esta razón, la distribución de la especie en el Parque permanece dudosa.

Es importante destacar que el ecosistema que se desarrolla en el margen rocoso del Río Uruguay presenta una dinámica particular, debido a que el mismo se encuentra sujeto a constantes cambios en el nivel que alcanza el agua y la velocidad de la corriente, en función del régimen estacional de precipitaciones y la actividad de represas hidroeléctricas instaladas en su cause superior (Coccoza Espósito 2004). Durante el año 2014, mientras se realizaba uno de los muestreos, se registró una importante crecida del Río Uruguay, que llegó a elevar su cota a más de 35 mts y provocó la desaparición de gran parte de las especies de plantas que se hallaban en el lugar. Se efectuó un seguimiento (actualmente en continuidad) de este ambiente para evaluar la recuperación de las poblaciones y la dinámica de la sucesión.

Se realizó una comparación florística respecto al Parque Estadual do Turvo (Brasil), área protegida que limita con el Parque Provincial Moconá (Fig. 1). Si bien en la actualidad no existen trabajos que compilen la flora de dicho Parque, a partir del hallazgo de información dispersa (Kuhnen et al. 2015; Inacio \& Jarenkow 2008) pudo observarse que ambas áreas poseen una baja cantidad de especies en común (Tab. S1, disponible en material complementario < https://doi.org/10.6084/ m9.figshare.9887627.v1>). En este sentido, se podrían realizar estudios exhaustivos sobre la presencia de ambientes y especies compartidas, con el objetivo de generar estrategias bilaterales de conservación de diversidad biológica entre Brasil y Argentina.

Finalmente, cabe destacar que para la flora argentina, de las 71 especies encontradas, 15 se distribuyen únicamente en la provincia de Misiones (Tab. S1, disponible en material complementario $<$ https://doi.org/10.6084/m9.figshare.9887627. v1>) y dos, Asplenium uniseriale y Serpocaulon catharinae, fueron citadas únicamente para el Parque Provincial Moconá (Meza Torres et al. 2010; Yañez et al. 2011). Esto pone de manifiesto la importancia estratégica que posee la Reserva de Biosfera Yabotí como reservorio de la biodiversidad de Argentina, la cual, junto con el Parque Nacional Iguazú, es uno de los principales relictos de Selva Paranaense que posee el país. Según la WWF (World Wild Life Fund for Nature) esta región forma parte de las 200 eco-regiones prioritarias para la conservación de hábitats terrestres, de agua dulce y marinos del planeta (Olson \& Dinerstein 2002). Los bosques subtropicales húmedos y mixtos que la componen, en los cuales solo subsiste el 7\% de su cobertura vegetal original, han sido clasificados como uno de los bosques con mayor diversidad biológica del 
mundo (Di Bitetti et al. 2003). Asimismo, Myers et al. (2000) los consideraron uno de los ocho "hot spots" más comprometidos a nivel mundial a partir de la pérdida de hábitats registrados. Este trabajo pone de manifiesto la relevancia que tiene la prospección de las áreas protegidas de Misiones con el fin de contribuir a la generación de futuras herramientas para la conservación de la flora nativa.

\section{Agradecimientos}

Los autores desean agradecer al cuerpo de guardaparques de la Reserva de Biosfera Yabotí por su asistencia durante la visita al Parque Provincial Moconá y, en particular, al Sr. Federico Castía por su excelente predisposición y asistencia logística. Al Ministerio de Ecología de la Provincia de Misiones por proporcionar los permisos de colección y a los curadores de los herbarios BA, LP y SI por proporcionar el material de estudiado. Este trabajo está dedicado a una mujer desconocida que marcó nuestro paso por el Parque.

\section{Referencias}

Arana MD \& Øllgaard B (2012) Revisión de las Lycopodiaceae (Embryopsida, Lycopodiidae) de Argentina y Uruguay. Darwiniana 50: 266-295.

Biganzoli F \& Múlgura de Romero ME (2004) Inventario Florístico del Parque Teyú Cuaré y alrededores (Misiones, Argentina). Darwiniana 42: 1-24.

Bertolini MP (1999) Plan de Manejo del Parque Provincial Moconá. Ministerio de Ecología y Recursos Naturales Renovables, Gobierno de la Provincia de Misiones. 121p.

Bolòs OD, Cervi AC \& Hatschbach G (1991) Estudios sobre la vegetación del estado de Paraná (Brasil meridional). Collectanea Botanica 1991: 79-182.

Cabrera AL \& Willink A (1973) Biogeografía de América Latina. Monografía 13, Serie de Biología. OEA, Washington D.C. $117 \mathrm{p}$.

Céspedes G \& Mereles F (2006) Estudio de la vegetación y la flora en el área de Aguará Ñú, Reserva de la Biosfera del bosque Mbaracayú, Paraguay Oriental. I. Rojasiana 7: 153-164.

Coccoza Espósito C (2004) Parque Provincial Moconá: diagnóstico para sudesarrollo turístico. Tesis de licenciatura en Turismo. Universidad Abierta Interamericana, Buenos Aires. 119pg.

Costa Assis F \& Salino A (2011) Dennstaedtiaceae (Polypodiopsida) no estado de Minas Gerais, Brasil. Dennstaedtiaceae (Polypodiopsida) in Minas Gerais, Brasil. Rodriguésia 62: 11-33.

Daviña JR, Honfi AI, Seijo GJ, Insurralde I \& Guillen $\mathrm{R}$ (1999) Floristic studies of the Moconá Park, Misiones, Argentina. Candollea 54: 231-249.

Daviña JR, Rodriguez E, Honfi AI, Seijo GJ, Insurralde
I, Guillen R \& Dematteis M (2003) Contribución al conocimiento florístico del Parque Provincial Moconá (Misiones, Argentina). Editorial Universitaria De Misiones, Posadas. 64p.

Di Bitetti MS, Placci G \& Dietz LA (2003) Una visión de biodiversidad para la Ecorregión del Bosque Atlántico del Alto Paraná: diseño de un paisaje para la conservación de la biodiversidad y prioridades para las acciones de conservación. World Wildlife Fund, Washington, D.C. 154p.

Fontana JL (1998) Análisis sistemático-ecológico de la flora del sur de Misiones (Argentina). Candollea 53: 211-300.

Giudice GE (1999) Sinopsis de las especies argentinas del género Adiantum (Pteridaceae, Pteridophyta). Darwiniana 37: 279-300.

Hirai RY \& Prado J (2000) Selaginellaceae Willk. in the state of São Paulo, Brazil. Brazilian Journal of Botany 23: 313-339.

Hirai RY \& Prado J (2012) Criptógamos do Parque Estadual das Fontes do Ipiranga, São Paulo, SP, Brasil. Pteridophyta: 1. Aspleniaceae. Hoehnea 39: 85-93.

Inácio CD \& Jarenkow JA (2008) Relações entre a estrutura da sinúsia herbácea terrícola e a cobertura do dossel em floresta estacional no Sul do Brasil. Revista Brasileira de Botânica 31: 41-51.

Kuhnen CFC, Marcolan DC \& Carvalho da Rocha M (2015) Proposta de educação ambiental na Unidade de Conservação Parque Estadual do Turvo, Derrubadas - Rio Grande do Sul. Revista Eletrônica em Gestão, Educação e Tecnologia Ambiental 19: 88-96.

Marquez GJ, Giudice GE \& Ponce M (2006) Pteridofitas de la Reserva "Valle del Arroyo Cuñá Pirú" (Misiones, Argentina). Darwiniana 44: 108-126.

Marquez GJ \& Yañez A (2012) Helechos epífitos de Alsophila setosa Kaulf. (Cyatheaceae, Pteridophyta) en la provincia de Misiones, Argentina. Boletín de la Sociedad Argentina de Botánica 47: 435-442.

Marquez GJ, Yañez A \& Kelly SJ (2016) Sobre la presencia de Megalastrum substrigosum (Dryopteridaceae) en Argentina. Boletín de la Sociedad Argentina de Botánica 51: 741-744.

Martínez OG (2003) Morfología esporofítica y revisión sistemática del complejo Pteris cretica (Pteridaceae-Pteridophyta) en América. Tesis Doctoral. Universidad Nacional de Salta, Salta. $172 p$.

Meza Torres EI, Keller H \& Brussa C (2010) New records of ferns from Northeastern Argentina and Uruguay. American Fern Journal 100: 183-187.

Moran RC (1995) Dryopteridaceae. En: Davidse G, Sánchez MS \& Knapp S (eds.) Flora Mesoamericana. Vol. 1. Universidad Nacional Autónoma de México, México DF. Pp. 210-218.

Moran RC, Prado J \& Labiak PH (2009) Megalastrum (Dryopteridaceae) in Brazil, Paraguay and Uruguay. 
American Fern Journal 99: 1-44.

Morrone JJ (2001) Biogeografía de América Latina y el Caribe. M\&T - Manuales \& Tesis SEA. Vol. 3. Editorial, Zaragoza. 148p.

Myers N, Mittermeier RA, Mittermeier CG, da Fonseca GAB \& Kent J (2000) Biodiversity hotspots for conservation priorities. Nature 403: 853-858.

Mynssen CM (2011) Woodsiaceae (Hook.) Herter (Polypodiopsida) no estado Rio Grande do Sul, Brasil. Pesquisas Botânica 62: 273-297.

Olson DM \& Dinerstein E (2002) The Global 200: priority ecoregions for global conservation. Annals of the Missouri Botanical Garden 89: 199-224.

Peña-Chocarro MC, Jiménez B, Marín G \& Knapp S (1999) Checklist of the pteridophytes of the Mbaracayú Forest Nature Reserve, Paraguay. Fern gazette 15: 221-258.

Ponce MM (1987) Revisión de las Thelypteridaceae (Pteridophyta) argentinas. Darwiniana 28: 317-390.

Ponce MM (1994) Pteridofitos. En: Kiesling R (ed.) Flora de San Juan. Vol. 1. Vazquez Manzini Editores, Buenos Aires. Pp. 17- 39.

Ponce MM (1998) Nuevas citas de Thelypteris subg. Amauropelta (Thelypteridaceae, Pteridophyta) para la flora de Argentina. Darwiniana 35: 177-178.

Ponce MM \& Arana MD (2016) Licofitas. Helechos. Gymnospermae. En: Zuloaga FO \& Belgrano MJ (eds.) Flora vascular de la República Argentina. Vol. 2. Estudio SIGMA S. R.L., San Isidro. 464p.

Ponce M, Mehltreter K \& de la Sota E (2002) Análisis biogeográfico de la diversidad pteridofítica en Argentina y Chile continental. Revista Chilena de Historia Natural 75: 703-717.

Ponce MM, Martinez OG \& Novara L (2012) Dryopteridaceae. Aportes Botánicos de Salta-Serie Flora 11: 1-31.

PPG I (2016) A community-derived classification for extant lycophytes and ferns. Journal of Systematics and Evolution 54: 563-603.

Prado J (2004) Criptogamos do Parque Estadual das Fontes do Ipirabga, São Paulo, Pteridophyta: 5. Dennstaedtiaceae. Hoehnea 31: 11-22.

Prado J \& Hirai RY (2008) Criptógamos do Parque Estadual das Fontes do Ipiranga, São Paulo, SP. Pteridophyta: 13. Lycopodiaceae e 20. Selaginellaceae. Hoehnea 35: 543-552.

Prado J \& Hirai RY (2010) Criptógamos do Parque Estadual das Fontes do Ipiranga, São Paulo, SP. Pteridophyta: 4. Davalliaceae, 19. Schizaeaceae,
23. Vittariaceae e 24. Woodsiaceae. Hoehnea 37: 791-800.

Prado J \& Windisch PG (2000) The genus Pteris L. (Pteridaceae) in Brazil. Boletim do Instituto de Botânica 13: 103-199.

Sakagami C (2006) Pteridófitas do Parque Ecológico da Klabin, Telêmaco Borba, Paraná, Brasil. Tese de Doutorado. Universidade Federal do Paraná, Curitiba. 200p.

Schwartsburd PB \& Labiak PH (2007) Pteridófitas do Parque Estadual de Vila Velha, Ponta Grossa, Paraná, Brasil. Hoehnea 34: 159-209.

Sehnem A (1968) Aspleniaceas. En: Reitz R (ed.) Flora Ilustrada Catarinense I. Herb. Barbosa Rodrigues, Itajaí. Pp. 1-96.

Sehnem A (1972) Pteridáceas. En: Reitz R (ed.) Flora Ilustrada Catarinense I. Herb. Barbosa Rodrigues, Itajaí. Pp. 1-244.

Sehnem A (1979) Aspidiáceas. En: Reitz R (ed.) Flora Ilustrada Catarinense I. Herb. Barbosa Rodrigues, Itajaí. Pp. 1-355.

Sobral M, Jarenkow JA, Brack P, Irgang B, Larocca J \& Rodrigues RS (2006) Flora arbórea e arborescente do Rio Grande do Sul, Brasil. RiMA, São Carlos. $359 \mathrm{p}$.

Sota de la ER (1960) Polypodiaceae y Grammitidaceae argentinas. Opera Lilloana 5: 1-229.

Sota de la ER \& Mickel JT (1969) Sinopsis de las especies argentinas del género Anemia Swartz (Schizaceae). Revista del Museo de La Plata, Sección Botánica 11: 133-152.

Sota de la ER (1977) Pteridophyta. En: Cabrera AL (ed.) Flora de la Provincia de Jujuy, República Argentina. Colección de Ciencias, Instituto Nacional de Tecnología Agropecuaria 13: 1-275.

Tryon RM \& Stolze RG (1989) Pteridophyta of Peru. Part II. 13. Pteridaceae-15. Dennstaedtiaceae. Fieldiana Botany 22: 1-127.

Tryon RM \& Stolze RG (1991) Pteridophyta of Peru, Part IV 17 Dryopteridaceae. Fieldiana Botany New Series 27: 1-176.

Tressens SG, Keller HA \& Revilla V (2008) Las plantas vasculares de la Reserva de uso múltiple Guaraní, Misiones (Argentina). Boletín de la Sociedad Argentina Botánica 43: 273-293.

Yañez A, Marquez GJ \& Ganem A (2011) Asplenium uniseriale (Aspleniaceae): una nueva cita para la flora argentina y novedades respecto a su hábito. Boletin de la Sociedad Argentina de Botánica 46: 355-359. 